\title{
The impact of COVID policies on the vitreoretinal service in St Paul's Eye Unit, Liverpool
}

\author{
Nikolaos Dervenis $\mathbb{1}^{1} \cdot$ Heinrich Heimann ${ }^{1} \cdot$ Rumana N. Hussain ${ }^{1} \cdot$ Paul Y. Chua ${ }^{1} \cdot$ Teresa Sandinha $^{1} \cdot$ Natàlia Vilà $^{1} \cdot$ \\ Ian Pearce ${ }^{1} \cdot$ Carl Groenewald ${ }^{1} \cdot$ Shi Zhuan Tan ${ }^{1}$
}

Received: 30 June 2020 / Revised: 13 July 2020 / Accepted: 15 July 2020 / Published online: 24 July 2020

(C) The Royal College of Ophthalmologists 2020

\section{To the Editor:}

Wickham et al. recently reported the impact of COVID-19 policies on the acute ophthalmology services in Moorfields and found the number of rhegmatogenous retinal detachment (RRD) presentation had fallen by $62 \%$ over 4 weeks since the implementation of lockdown measures on the 23/3/2020 in the UK [1].

The St Paul's Eye Unit, a tertiary referral centre in Liverpool, have similarly conducted a prospective audit over a 12-week period from $23 / 3 / 2020$ to $14 / 6 / 2020$ to investigate firstly, the impact of COVID-19 on our emergency vitreoretinal service and secondly, the effect of COVID-19 policies on individual's decision to seek medical attention, through a pre-designed questionnaire completed by patients at presentation.

The baseline characteristics of our cohort are presented in Table 1. Similar to the Moorfields' experience, we observed a fall of $58 \%$ in RRD cases over the study period $(n=76)$ compared to the period from $1 / 12 / 2019$ to $29 / 2 / 2020$ ( $n=$ 176). We did not observe a significant change in number of cases despite easing of lockdown measures first on the $13 / 5 / 2020$ and secondly on the $1 / 6 / 2020$.

Overall, $13(17.1 \%)$ patients had COVID-related concerns leading to late presentation and 3 out of 13 had more than one concerns. Of these, six were self-isolating, five were unsure if the ophthalmology service was running and four were concerned about contracting COVID-19. Only 1 out of 76 patients was COVID positive on swab (asymptomatic) and none contracted COVID-19 during the followup period. The median delay in presentation was 10 days

Nikolaos Dervenis

nikosdervenis@gmail.com

1 St Paul's Eye Unit, Royal Liverpool University Hospital, Liverpool, UK (range 3-40). The results of the questionnaire are presented in Table 2.

Our study showed that there is a significant drop in RRD cases since lockdown. Although only $17 \%$ reported delayed presentation due to COVID-19 policies, it remains unclear whether a proportion of patients especially those shielding, are still yet to access the service and this will become clearer as the weeks progress. It is also reassuring that none of the patients contracted COVID-19 during the study period. These were short-term findings and longerterm collaborative audit with other centres will help determine the actual impact of COVID-19 on our service and patients.

Table 1 Baseline characteristics.

\begin{tabular}{ll}
\hline Baseline characteristics & Total $n=76$ \\
\hline Age (mean \pm SD) & $61( \pm 12.6)$ \\
Sex, $n(\%)$ & $29(38.2)$ \\
$\quad$ Female & $47(61.8)$ \\
$\quad$ Male & \\
Retinal detachment, $n(\%)$ & $63(82.9)$ \\
$\quad$ Primary & $13(17.1)$ \\
$\quad$ Secondary detachment & \\
Macula status, $n(\%)$ & $45(59.2 \%)$ \\
$\quad$ Macula on & $31(40.8 \%)$ \\
$\quad$ Macula off & \\
Baseline best-corrected visual acuity (logMAR) & -0.18 to 2.70 \\
Range & $0.78(0.89)$ \\
$\quad$ Mean \pm SD & \\
Ocular co-morbidities, $n(\%)$ & $26(34)$ \\
Myopia (>4D) & 0 \\
Amblyopia & $4(5.3)$ \\
Glaucoma & $4(5.3)$ \\
Macular pathology & \\
\hline
\end{tabular}


Table 2 Results of questionnaire.

Questions

Answers, $n(\%)$

Has COVID-19 lockdown led to you presenting late to the service?

Yes

No

Not sure

If yes, what was the reason for late presentation? Symptomatic with COVID

Yes

0

No

Advised to self-isolate

Yes

No

$70(82.1)$

Self-isolating as family member was symptomatic

Yes

0

No

Carer for vulnerable person

Yes

$4(5)$

No

Concerned about getting infected

Yes

No

Unsure if ophthalmology service is running

Yes

$5(6.6)$

No

The service contacted was not running (e.g. community optometrist)

Yes

No

$74(97.4)$

Other reasons

'Thought symptoms related to glaucoma and appointment was cancelled' 2 (2.6)

'Wife is isolating'

$1(1.3)$

\section{Compliance with ethical standards}

Conflict of interest The authors declare that they have no conflict of interest.

Publisher's note Springer Nature remains neutral with regard to jurisdictional claims in published maps and institutional affiliations.

\section{References}

1. Wickham L, Hay G, Hamilton R, Wooding J, Tossounis H, da Cruz $\mathrm{L}$, et al. The impact of COVID policies on acute ophthalmology services-experiences from Moorfields Eye Hospital NHS Foundation Trust. Eye. 2020:34;1189-92. 